\title{
Decision support for multi-component systems: visualizing interdependencies for predictive maintenance
}

\author{
Milot Gashi \\ Pro2Future GmbH Graz, Austria \\ milot.gashi@pro2future.at
}

\author{
Belgin Mutlu \\ Pro2Future GmbH Graz, Austria Graz University of Technology, Austria \\ belgin.mutlu@pro2future.at lindstaedt@tugraz.at \\ Stefan Thalmann \\ University of Graz, Austria \\ stefan.thalmann@uni-graz.at
}

\begin{abstract}
Taking dependencies between components seriously and considering the multi-component perspective instead of the single-system perspective could help to improve the results of predictive maintenance $(P d M)$. However, modeling and identifying the interdependencies in complex industrial systems is challenging. A way to tackle this challenge and to identify interdependencies is using visualization. To the best of our knowledge, existing research on visualizing interdependencies is not applied to multi-component systems (MCS) so far. Further, it is not clear how visualization approaches can provide suitable decision support to identify interdependencies in PdM tasks. We evaluate three key visualization approaches to represent interdependencies in the context of PdM for MCS using a crowd-sourced design study in a questionnaire survey involving 530 participants. Based on our study, we were able to rank these approaches based on performance and usability for our given PdM task. The multi-line approach outperformed other approaches with respect to performance.
\end{abstract}

\section{Introduction}

Predictive Analytics, and especially PdM has gained vast attention in recent years in both research and digitized industry. PdM provides many advantages on increasing productivity and reliability and is the most known use case for data-driven decision support in industrial settings [1]. In recent years, huge advances were made in the development of predictive models, thus improving decision support. However, current studies reveal that the industry is not yet satisfied with PdM-solutions [2,3]. This is mainly as a result of mass customization, more complex production processes, and shorter product life cycles in digitized manufacturing
[2]. Traditional PdM approaches consider even very complex industrial systems on a single component level (e.g., system-level or a component) without paying enough attention to the interdependencies of components [4]. MCS seem promising in this regard as they explicitly consider interdependencies between system components and thus promise more accurate and understandable models [5, 4]. For instance, an old worn-out component that interacts with new components will potentially accelerate the wear rate of a new one. This potential information will improve the prediction results and maintenance scheduling. However, identifying and modeling interdependencies in complex industrial systems is not a trivial task and currently, the major barrier of introducing MCS in the industry [6].

MCS is defined as a system that consists of multiple components and these components strongly interact with each other. In this case, a type of interdependencies (i.e., interactions) is deterioration effect between components. Modeling and interpreting interdependencies provide many advantages, such as more accurate results, higher interpretability, higher acceptance rate of these models, and finally improved maintenance scheduling strategies $[5,7,4]$. Until now some initial research attempting to improve the decision-making process for PdM in the context of MCSs is conducted [7, 5]. In these works, the main focus is to provide decision support to shop floor workers by providing the most suitable predictive maintenance policy. Mostly the interdependencies are defined based on domain expert knowledge due to the lack of amount of data required to represent an MCS.

One important aspect of decision support in context of MCS, however, is the presentation of interdependencies to end-users. One way to present the interdependencies in an appropriate and user-friendly way are visualization approaches [8]. In literature, some studies have used different visualizations to present 
the results in the context of MCS, even though the visualization of interdependencies was not the focus of these studies $[9,10,11,12,13]$. To the best of our knowledge, we did not find any approach tackling this particular challenge of visualizing interdependencies for MCSs. Although, the potential to understand and identify interdependencies will provide a basis to build a vision for a predictive model, which we lack because of the missing interdependencies.

In this paper, we aim to explore how do visualizations help the users to identify the interdependencies within an MCS. Moreover, we aim to identify cost-optimized oriented approaches. We also aim to rank visualizations in terms of usability. We conducted a crowd-sourced study involving 530 participants, where we were able to identify the best approach regarding the performance.

\section{Theoretical background}

\subsection{Multi-component systems and interdependencies}

MCS view in the context of PdM helps to improve predictive results, as well as understanding and interpretation of the predictions, thus, enhancing maintenance scheduling and decision support $[9,4]$. Current research has shown that interdependencies between components exist and have a strong impact on the deterioration process, as well as on the economical aspect $[14,4,9]$.

In the existing literature, interdependencies between components are grouped into different categories: stochastic, economic, and structural interdependencies. First, stochastic interdependencies represent the deterioration effect between components within a system. For instance, a worn-out component will accelerate the wear-out rate of other components that are interacting within an MCS. Second, economic interdependencies focus directly on the generated costs, respectively, reduced costs that can be assured through performed maintenance of components together, rather than maintaining separately. Finally, structural interdependencies describe the effect that components cause on each other, in case that these components are structurally coupled with each other. The effect can be in costs, as well as on the deterioration rate [14]. For instance, Rasmekomen et al. [14] analyzes the structural effect based on the deterioration rate. The presence of various interdependencies within MCSs increases the complexity further. Therefore, to build human-centric PdM solutions, decision support to identify and model these aspects is required.

In practice, decision support is crucial to provide efficient, faster, and reliable alternatives in the decision-making process. A number of research results of decision support for industry-based PdM have emerged in literature $[15,16,17,18,19,20]$. These tools have enabled decision support providing reasonable results by incorporating the data-driven approaches. For example, Yam et al. [17] developed a PdM decision support system (DSS) based on a recurrent neural network. Moreover, We et al. [16] introduced an integrated neural network based on a decision support system for predictive maintenance of rotational products. These works aim to assist shop floor workers in the decision-making process by providing recommendations regarding the maintenance actions or selection of the most suitable analytical approach for PdM solutions. Initially, this works focuses on a single component system (e.g., system-level or a component) providing acceptable results. However, the complexity of systems in digitized manufacturing increases due to mass customization and shorter product life cycles in digitized manufacturing [2], or the presence of different variants and configurations within one machine [4]. This leads to the need for new solutions to handle the complexity and its challenges properly. MCS view in context of PdM seems promising in this regard. However, in the past, the lack of data and the degree of complexity present within MCSs prevented sufficient research on the implementation and application of MCS perspective [4]. Nevertheless, with the increase of the information basis (growing availability of cheap and powerful sensor technology [21]) and huge advances in AI and data processing capabilities [22], research in MCSs seems feasible. As a result, this could help to reduce maintenance costs and improve the quality of products.

\subsection{Visualization for decision making}

Data visualization is a powerful representation of the information in decision-making process. It can accelerate perception, provide awareness and control, and harness knowledge to gain a competitive advantage in making valuable decisions [15, 23, 24]. Current research has shown that data visualization helps to enhance and provide more effectiveness and control during the maintenance process $[25,26,27]$. In particular, it provides a better understanding of the data and faster knowledge acquiring for shop floor workers. The main focus of these works has been towards maintenance actions $[27,25,26]$. In this regard, the aim is to visually help the shop floor workers to understand and improve the performance of the maintenance process. 
In literature, you can find many examples where visualization for decision support in the context of PdM has been applied [28, 29, 30, 16]. In general, different parameters (e.g., sensor data) are visualized, thus, providing a possibility for visual condition monitoring from the shop floor workers. This helps to understand and interpret better the predictive results. Moreover, visualizations have been applied for PdM in context of MCSs to enhance decision-making process. In this case, the aim is not only to show the deterioration of the component over time, but also the interdependencies effect between the components. Extensive research works focusing on PdM in the context of MCSs, which used visualization to demonstrate the presence of interdependencies can be found $[9,10,11,12$, 13]. For instance, Assaf et al. [9] used line charts to show the presence of interdependencies between components. As a result, a mathematical model for multi-component degradation modeling is presented. Shahraki et al. [10] used multi-line visualizations to show interdependencies between the components within an MCS. However, none of these studies has evaluated the usefulness of visualization aiming to show and identify the interdependencies.

\section{Interdependence's visualization approaches}

In the literature, we identified potential candidate approaches suitable for modeling interdependencies using visualization approaches: line-based approach [9], matrix-based approach [31, 11], multi-line approach [32], bar-based approach [13], and stacked area approach [33]. Next, after multiple iterations of discussion with domain experts, we defined the selection rule to drill down to the relevant approaches: First, we selected visual approaches that highlighted interdependencies over time. Second, we considered visual approaches that emphasize the performed component replacements e.g., maintenance actions. Finally, space reduction is considered as an important aspect to select these approaches. For example, the multi-line approach requires less space compared to the line chart approach where each component is visualized in a separate chart. As a result, we narrow down to three important approaches: The multi-line, the heatmap (adaption of matrix-based approach), and the stacked-area approach.

\subsection{Multi-line approach}

Multi-line approaches are known as important approaches for robust visualization of multiple time-based data e.g., time series [32]. The multi-line approach is appropriate for pattern recognition and relationship analysis. In our specific case, we are interested in the visualization of interdependencies between components which deteriorate over time. In this context, the multi-line approach meets exactly the requirements for visualizing MCS interdependencies. Hence, we consider multi-line (see Figure 1) for further exploration within our study.

Multi-line approach is composed of two axes: $\mathrm{x}$-axis representing the distance information in $\mathrm{km}$ and $y$-axis representing the deterioration time, whereby 1 represents a fully worn-out component, respectively, 0 a completely new component. Deterioration as a physical parameter evolves over the distance as shown in Figure 1. Deterioration switches to 0 , when a maintenance activity is performed and the component is replaced. Moreover, a single line represents the deterioration state of a specific component.

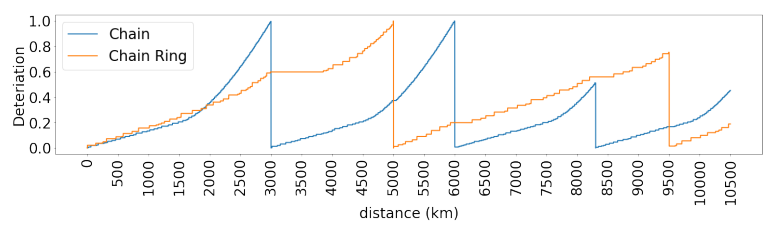

Figure 1: MCS interdependencies presented using multi-line visualization approach. X-Axis representing the distance information in $\mathrm{km}$ and $\mathrm{y}$-axis representing the deterioration time.

\subsection{Heatmap approach}

The Heatmap approach visualizes data using table format (matrix) and a variety of colors [31]. In this case, the possibility to apply different variables in the rows and columns provides an opportunity for cross-examining multivariate data. Moreover, the coloring of the cells within the table represents the magnitude of the variable at a specific state. This provides an opportunity for showing variance across multiple variables. Heatmaps are valuable, when aiming to recognize patterns or estimating the similarity within multivariate data. Heatmaps require less space compared to other visual approaches, thus, are suitable for visual feature reduction challenge.

A Heatmap approach is a visual representation of components deterioration data over the distance, where the individual values within the matrix are represented as colors. A row contains deterioration state information for a single component. Whereby, the $\mathrm{x}$-axis of the heatmap-matrix 2 represents the distance information in $\mathrm{km}$. The color within a specific row encodes the corresponding deterioration state of the component. A 
white color reveals a new component, whereby a black color reveals a fully worn-out component.

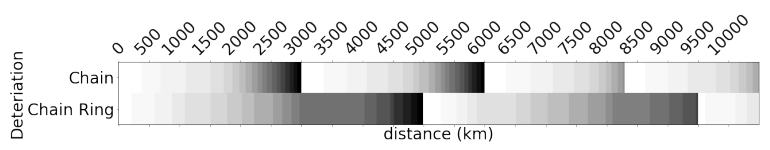

Figure 2: MCS interdependencies presented using the heatmap visualization approach. X-Axis representing the distance information in $\mathrm{km}$. The color within a specific row represents the corresponding deterioration state of the component. A white color reveals a new component, whereby a black color reveals a fully worn-out component.

\subsection{Stacked-area approach}

Stacked-area approaches are visualization approaches for representing multiple time-series by stacking filled shapes that represent single time-series on top of each other [34]. The high of each stream at a specific time point represents its value. The stacked-area approach is suitable for pattern recognition, comparison, and causal analysis. These characteristics fit exactly to challenge of interdependencies recognition for MCS.

Stacked-area approach (shown in Figure 3) is composed of two axes: the $\mathrm{x}$-axis representing the distance information in $\mathrm{km}$ and the $\mathrm{y}$-axis representing the deterioration time. In reality, for a single component, 1 represents a worn-out component and 0 an unused component. Important is to note, that the stacked-area, in contrast to the multi-line approach, accumulates the deterioration state of components (e.g., two components). For example, if both components are fully worn-out, the upper limit will be 2 . This could increase the probability for distortion while reading and comparing values within the stacked-area [35].

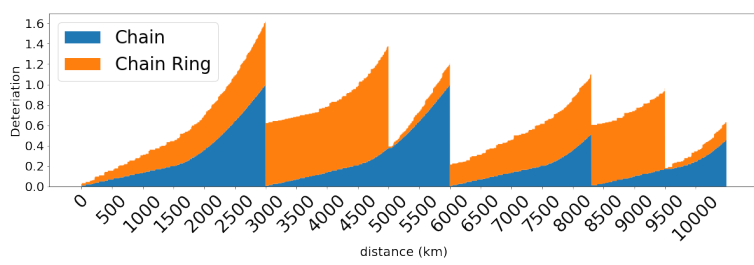

Figure 3: MCS interdependencies presented using stacked-area visualization approach. X-Axis representing the distance information in $\mathrm{km}$ and $y$-axis representing the deterioration time.

\section{Evaluation study}

In this section, we investigate different visualization approaches concerning interdependencies where the aim is to evaluate (i) how do the visualization helps the users to identify the interdependencies within MCSs, (ii) which visualization/-s is/are the most appropriate ones for visualizing and identifying the interdependencies. To do so, we design a study on a crowd-sourced platform to elicit preferences and user performance using these approaches. This section describes in detail the data sources, the method, and the metrics used within this study.

\subsection{Use case and data description}

As industrial machines are very complex and specific, we needed an MCS use case, which is common and most people are aware of and understand it. For this purpose, we decided to select a bicycle as a suitable example for simple MCS. The bicycle consists of a small number of components and these components strongly interact with each other. Moreover, the bicycle example provides a good basis to demonstrate the results in an understandable way. Furthermore, the access to subjects is simpler compared to a real industrial use case. On the other hand, the knowledge collected within this use case is theoretically transferable to a real industrial setting. In this context, we focus on two specific components: chain and chain-ring. Domain experts (i.e., bicycle mechanic) defined these two components, as components which introduce a strong level of interdependencies.

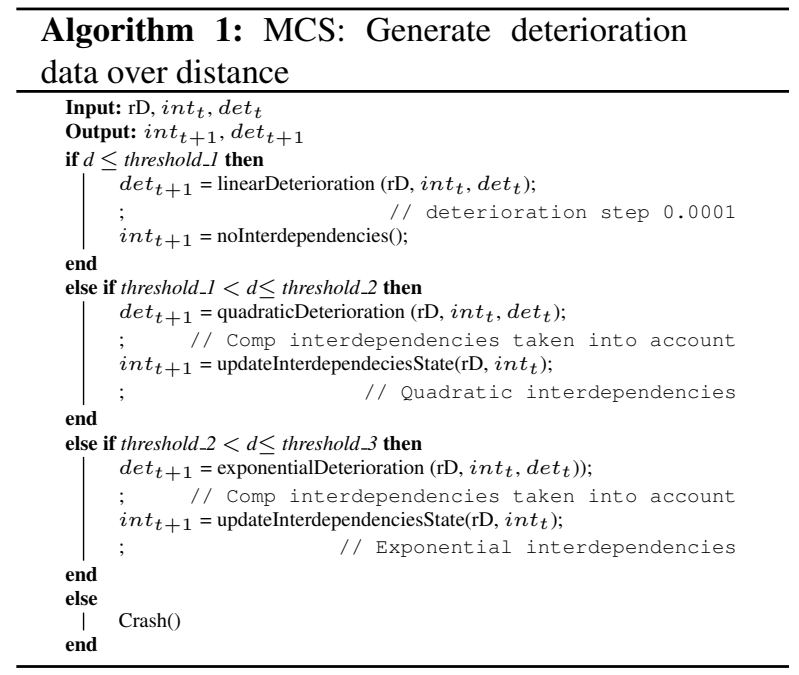

Due to missing condition monitoring data for specific components (e.g., chain and chain-ring), we 
have resorted synthetic data. Based on the description of the deterioration time and deterioration effect between components we generated health description data. The description of the component aging is provided by domain experts through multiple interviews. This helped us to generate data close to reality in a standardized and simpler way. Based on the interview results we defined different assumptions: (1) chain crashes after $4000 \mathrm{~km}$ (i.e., threshold_3), (2) chain-ring crashes after $10000 \mathrm{~km}$ (i.e., threshold_3), (3) if a chain component has been in use less than $1500 \mathrm{~km}$ i.e., threshold_1, then no deterioration effect toward other components is present, (4) if chain-ring has been in use for less than $4000 \mathrm{~km}$ (i.e., threshold_1), then no deterioration effect toward other components is present. (5) if chain has been in use more than $3000 \mathrm{~km}$ (i.e., threshold_2), the deterioration rate and effect is exponential. (6) if chain-ring has been in use more than $8000 \mathrm{~km}$ (i.e., threshold_2), the deterioration rate and effect is exponential.

As a result, a mathematical model taking into account the corresponding parameters (e.g., chain's threshold_1) for every relevant component (i.e., chain and chain-ring) is designed (see algorithm 1). The mathematical model is used to generate the deterioration data concerning interdependencies. This provides two different advantages: (1) the model help us to generate synthetic data based on the domain experts, thus leading to a data-set that describes a system behavior for bikers. (2) This model will be used to evaluate the results during a design study, thus increasing the plausibility of collected data.

Let $\operatorname{ch} X_{t}$ and $\operatorname{chr} X_{t}$ represent the accumulated deterioration of chain at $t$ distance [km], respectively, deterioration of chain-ring. In such cases, deterioration state (e.i., $\operatorname{ch} X_{t}$ and $\operatorname{chr} X_{t}$ ) is updated for each deterioration step (e.i., $t$ ). In our case, a deterioration step represent 1 meter $(\mathrm{m})$ ride of the bike (i.e., $r D$ ). At each deterioration step, the deterioration of the chain and chain-ring is evaluated using the algorithm 1. Algorithm 1 takes as input the relative distance (i.e., $r D$ ), current interdependencies effect (i.e., int $_{t}$ ), and current deterioration state of the corresponding component (i.e., $\left.\operatorname{det}_{t}\right)$. As a result, the deterioration state of the component (i.e., $\operatorname{det}_{t}+1$ ) and the interdependencies effect (i.e., $i n t_{t}+1$ ) for the next step is estimated and provided as output. In particular, the deterioration could evolve in three different forms (depending on the relative distance): linear (LinearDeterioration (...)), quadratic (QuadraticDeterioration (...)), or exponential (ExponentialDeterioration (...)) form.

For the design study, two different scenarios are considered. We use these two data-sets to generate the scenarios for the visualization approaches. This is crucial as it helps to compare these approaches and interpret the results.

- Scenario 1 is a data-set that represents the deterioration of chain and chain-ring over a distance. Multiple replacements are performed randomly for each of the components. In this data-set, the deterioration effect is considered and modeled based on the algorithm 1 .

- Scenario 2 is a data-set where deterioration of the components over distance is modeled. Similar to scenario 1 , this data-set models deterioration of components where randomly multiple component replacements are performed on both chain and chain-ring. In particular, this data-set demonstrates the case which leads to a crash. On the one hand, the deterioration effect (interdependencies) is clearly shown. On the other hand, this data-set is terminated with a crash, where one of the components is overused, thus, requiring to replace both components.

\subsection{Procedure}

This design study aims to understand if users can identify and interpret interdependencies between components of an MCS represented with different visualization approaches. Moreover, we want to identify which visualization/-s is/are the most appropriate ones for visualizing and identifying the interdependencies of an MCS. For this purpose, a crowd-sourced study is designed to evaluate different visualization approaches. In the past, crowd-sourced studies showed significant results when comparing different visualizations [36, 37]. Each participant has to evaluate in detail only one specific approach. This decision will help to avoid biased data [38, 39]. In particular, to avoid biased data (i.e., retrospective bias [39]), we also randomly assigned the visualization approach to analyze in detail as well as the order of answers of all the questions within the design study.

First, a description and purpose of the study, altogether, with the information about the respect of confidentiality regarding the data is provided to each participant. Second, the participant is asked to answer some demographic questions regarding the expertise on visualization and education level. Further demographic data are provided directly from platform, which is used to conduct the study. Third, the MCS use-case and the definition of interdependencies is presented through a video animation.

Next, the participant performed the main task, i.e., 
he/she evaluated one of the three approaches in detail. The task was designed as follows: (1) a short description of the visualizations was shown to the participant, (2) two different scenarios of components deterioration over time (e.g., distance) and performed maintenance actions (e.g., replacements) using the corresponding visualization approach were shown to the participant. The participant is asked to analyze these scenarios in detail and try to identify the interdependencies between components. (3) Next, the participant is asked to rank the identified level of interdependencies. Furthermore, the participant is asked to design a maintenance strategy (e.g., define component replacements over the distance) for a distance ride of $10000 \mathrm{~km}$. Moreover, a limited budget of $600 €$ is provided altogether with the cost information of both components. Chain value was 20 $€$ and chain-ring $200 €$. This task is designed based on the suggestion from Kittur et al. [40] to motivate the participants to study the visualization in an accurate way and prevent random answers. Fifth, we performed a usability evaluation based on the System Usability Scale (SUS) [41]. Sixth, subjective feedback has been collected through a post-task questionnaire based on NASA TLX ${ }^{1}$ covering six dimensions of workload: mental demand, physical demand, temporal demand, effort, frustration, and perceived performance. Finally, to each participant, all three approaches are shown, and we asked them to select the approach that they would use to identify interdependencies between components.

\subsection{Evaluation protocol}

We conducted an offline evaluation of the collected results. First, we analyzed and compared the distribution of the data using both visualization tools as well as statistical approaches. As a visualization tool, we used bar charts with confidence intervals (known as error bars), providing two crucial pieces of information, the average of the data distribution and the confidence interval. This information helps us to estimate visually if two distributions are significantly different. On the other hand, the Mann-Whitney $U$ test [42] is used as a statistical approach to evaluate the difference of distributions. This approach provides more reliable results as it is non-parametric and is not dependent on normally distributed data. In this case, the significant differences with $\mathrm{p}<0.01$ are estimated. Second, we used the mathematical model introduced in 4.1, which is used to generate the data-sets. The model is used to evaluate if a provided strategy is suitable to keep the bicycle in operational state or will crash. A strategy that led to a crash is considered a failed, otherwise, a

\footnotetext{
${ }^{1}$ https://humansystems.arc.nasa.gov/groups/tlx/
}

successful strategy.

\section{Discussion of results}

\subsection{User study: data elicitation}

In this crowd-sourced study, 704 users participated. Whereby, $530(\mathrm{M}=435, \mathrm{~F}=84, \mathrm{~N} / \mathrm{A}=11)$ participants, age 18-65 are approved as shown in Table 5a. The strategies provided by participants are used to evaluate the quality of the submissions. For example, strategies that contained only random numbers are rejected, thus, considering only serious attempts. As a result, 72 submissions are rejected. Moreover, 89 users returned their submission i.e., results are not submitted. Finally, 13 participation are rejected from the platform, due to timed out, which was 45 minutes (m) by default. The participants had experience with visual- and data analytic tools. Moreover, all participants had experience in the industry and are well educated (529 participants with at least a bachelor's degree). Participants needed $\mu=12 \mathrm{~m}$ and 42 seconds (s) to successfully analyze the heatmap approach. Stacked-area visualization is successfully analyzed in $\mu=13 \mathrm{~m}$ and $42 \mathrm{~s}$ and the multi-line approach is successfully analyzed in $\mu=12$ $\mathrm{m}$ and $50 \mathrm{~s}$.

Finally, the participants provided subjective feedback with regard to the workload. The workload has been estimated using the results of the NASA TLX questionnaire. In general, the workload of all the approaches seems low (mean below 50): (heatmap) $\mu$ $=27.54 \sigma=6.21$, (stacked-area) $\mu=28.48 \sigma=5.99$, (multi-line) $\mu=26.78 \sigma=6.65$. Moreover, the results focusing on performance and frustration showed a significant difference. In this case, the Mann-Whitney $\mathrm{U}$ test showed that the distribution is significantly different with $\mathrm{p}<0.01$. In both cases, it is obvious, that the participants felt more confident while using multi-line approach and less frustrated. In contrast, the participants who evaluated stacked-area approach felt less confident and more frustrated during the evaluation.

The learning effect based on SUS is estimated, to evaluate the level of training needed for these approaches. In general, low learning effect has been revealed (mean score below 68): (heatmap) $\mu=63.92 \sigma$ $=27.82$, (stacked-area) $\mu=62.34 \sigma=25.35$, (multi-line) $\mu=65.3 \sigma=24.51$. In particular, we can find some significant differences between the approaches shown in Figures $4 \mathrm{a}$ and $4 \mathrm{~b}$. The significance of the distribution is shown using the bar charts with confidence interval and Mann-Whitney U test approach with $\mathrm{p}<0.01$.

These results show that participants analyzing heatmap and multi-line approaches were more 
confident, needed low overall workload, and most importantly the perception is that less training is needed to use these approaches. But this was not the case with stacked-area approach, where participants thought that this is less intuitive and felt less confident while analyzing this approach. Interestingly, the aforementioned results show a similar performance between the heatmap and multi-line approach. Even though, stacked-area and multi-line approaches are primarily designed for multiple time-series [34], the results indicate that they perform significantly differently while aiming to identify interdependencies for MCS. Therefore, a more detailed exploration of these approaches could help to explain further the similarity and dissimilarities between these approaches.

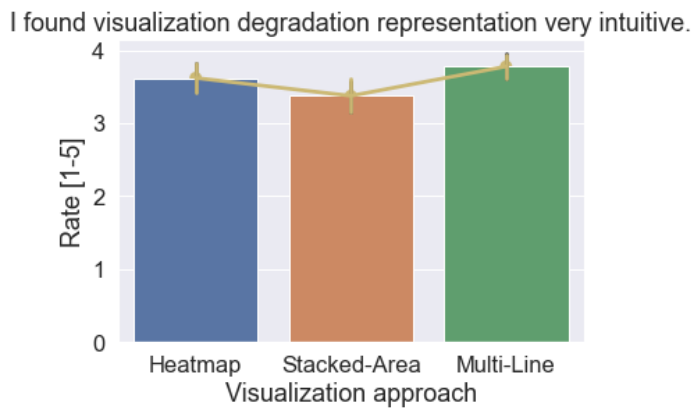

(a) Approache's perception

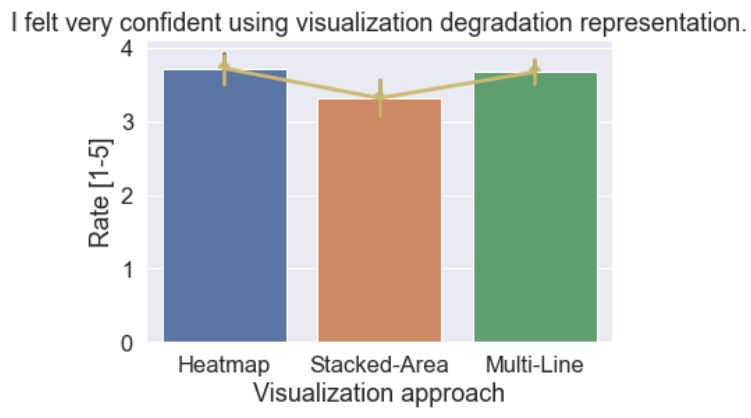

(b) Evaluation of the confidence level

Figure 4: Usability evaluation: A visual comparison of confidence and intuition level between the analyzed approaches.

\subsection{Strategy validation and cost optimization}

The approved 530 strategies are analyzed in detail. First, we validated the provided strategies (i.e., deterioration validation) based on mathematical model introduced in 4.1. Strategies that generated a crash, are considered as failed strategies, otherwise, are considered as a successful strategies (see Figure 5a). Overall, 334 strategies were successful and 197 strategies failed which resulted in a crash. In general, participants that analyzed multi-line approach provided a lower rate of failed strategies with only $31 \%$, followed by heatmap strategy with $37 \%$ of failed strategies. The participants using a stacked-area visualization returned a higher rate of failed strategies with $44 \%$. Along deterioration validation process a cost-based validation of the provided strategies (see Figure 5b) is conducted. Cost-based validation is applied on top of the deterioration validation results. In such cases, a strategy that exceeds the provided cost limit of (600 $€)$ is classified as failed, otherwise, as a successful strategy. As a result, the multi-line approach showed the lowest rate of failed strategies with $30 \%$, followed by heatmap approach with $37 \%$, respectively, $41 \%$ for the stacked-area visualization.

Additionally, we estimated the costs for each visualization approach with respect to the provided strategies (see Figure 6). The results showed that the participants analyzing stacked-area approach invested around $500 €$ on average. But, participants who analyzed heatmap and multi-line approach invested less than $400 €$ on average. These results were statistically significant based on the Mann-Whitney U test approach with $\mathrm{p}<0.01$.

The results from the strategy validation process indicate that multi-line approach showed a lower error rate. This suggests that multi-line could be the appropriate approach that shows a high degree of visual perception and recognition. Moreover, perception results for stacked-area approach were poorer compared to the other approaches. This could be due to distortion effects while aiming to read and compare values within stacked-area [35]. On the one hand, the cost optimization results suggest that heatmap and multi-line are suitable approaches. On the other hand, the stacked-area approach seems to be the more offensive approach, which could be helpful in more sensitive settings [43]. Usually, offensive approaches are appropriate in sensitive settings, where breakdown should be prevented due to safety or economic costs. Furthermore, the evaluation in this work was not exhaustive. In particular, we evaluated the interdependencies recognition, but we chose not to include semantic enrichment. Semantics enrichment allows identifying, extracting, and presenting the relevant interdependencies [44]. Last but not least, this is a complex task but is crucial for clear and precise knowledge understanding, documentation, and communication. 


\begin{tabular}{ccccc}
\hline \multicolumn{5}{c}{ Deterioration validation } \\
\hline $\begin{array}{c}\text { Visualization } \\
\text { type }\end{array}$ & $\begin{array}{c}\text { Successful } \\
\text { strategies }\end{array}$ & $\begin{array}{c}\text { Failed } \\
\text { strategies } \\
\text { (Crash) }\end{array}$ & $\begin{array}{c}\text { All } \\
\text { strategies }\end{array}$ & $\begin{array}{c}\text { Failed } \\
\text { strategies } \\
\text { (\%) }\end{array}$ \\
\hline Multiline & 127 & 58 & 185 & $\mathbf{0 . 3 1}$ \\
\hline Heatmap & 116 & 68 & 184 & $\mathbf{0 . 3 7}$ \\
\hline Stacked Area & 90 & 71 & 161 & $\mathbf{0 . 4 4}$ \\
\hline SUM & $\mathbf{3 3 3}$ & $\mathbf{1 9 7}$ & $\mathbf{5 3 0}$ & $\mathbf{0 . 3 7}$ \\
\hline
\end{tabular}

(a) Deterioration validation strategy process. A maintenance strategy that crashes within $10000 \mathrm{~km}$ is classified as failed, otherwise, successful strategy.

\begin{tabular}{ccccc}
\hline & \multicolumn{4}{c}{ Cost validation } \\
\hline $\begin{array}{c}\text { Visualization } \\
\text { type }\end{array}$ & $\begin{array}{c}\text { Successful } \\
\text { strategies }\end{array}$ & $\begin{array}{c}\text { Failed } \\
\text { strategies } \\
\text { (costs > 600) }\end{array}$ & $\begin{array}{c}\text { All } \\
\text { strategies }\end{array}$ & $\begin{array}{c}\text { Failed } \\
\text { strategies } \\
\text { (\%) }\end{array}$ \\
\hline Multiline & 126 & 54 & 180 & $\mathbf{0 . 3}$ \\
\hline Heatmap & 115 & 66 & 181 & $\mathbf{0 . 3 7}$ \\
\hline Stacked Area & 88 & 62 & 150 & $\mathbf{0 . 4 1}$ \\
\hline SUM & $\mathbf{3 2 9}$ & $\mathbf{1 8 2}$ & $\mathbf{5 1 1}$ & $\mathbf{0 . 3 6}$ \\
\hline
\end{tabular}

(b) Cost-based validation strategy process. A maintenance strategy that exceeded the provided limit of costs $(600 €)$ was classified as failed, otherwise, successful strategy.

Figure 5: Strategy validation process

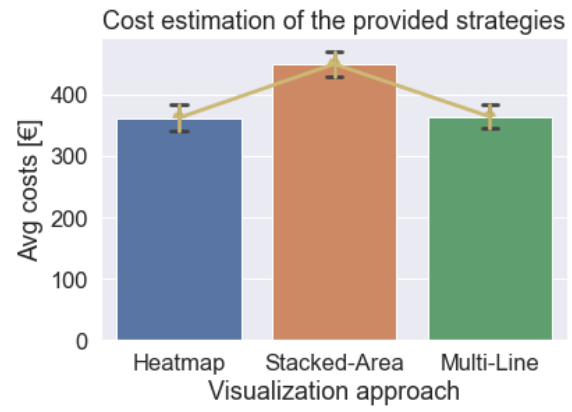

Figure 6: Average cost estimation: Heatmap and multi-line approach show significant results while aiming for cost optimization

\subsection{Preference transitions}

At the end of the design study, participants were familiar with one of the approaches. Finally, we showed to every participant all three approaches, and we asked them to select the approach that they think will help them most to identify interdependencies between components.

The results are shown in Figure 7. Whereby, $35 \%$ of all participants used the multi-line approach in the experiment. The majority of these participants preferred to keep using the multi-line approach (73\%). However, only a small number of participants preferred to switch to heatmap (11.5\%) or stacked-area approach (15.4\%). Moreover, 35\% of all participants used the heatmap approach. Even though several participants preferred to keep using the heatmap approach (45.5\%) for analyzing interdependencies, still, a reasonable number of participants preferred to switch to multi-line $(27.3 \%)$, respectively, stacked-area approach (27.2\%). Interestingly, the majority of participants who analyzed the stacked-area approach in detail preferred to switch to the multi-line approach (56.4\%). A small number of participants preferred to keep using the stacked-area approach $(29.1 \%)$, or to switch to the heatmap approach $(14.5 \%)$. As a result, $52.2 \%$ of participants preferred the multi-line approach. Whereby, $24.2 \%$ of participants preferred the heatmap approach and only $23.6 \%$ the stacked-area approach. These results show clear domination of the multi-line approach in contrast to the heatmap and stacked-area approach. This is an indicator that the multi-line approach could be appropriate for the recognition of interdependencies within an MCS. Although, clear causation of flowing tendencies toward the multi-line approach is not obvious from this study. This is a valid research question for future work as participants switch to all approaches. Relevant works $[45,46]$ show that users' characteristics such as cognitive abilities have an impact on the performance or subjective preferences with a given visualization approaches and thus also our three approaches might depend on user characteristics.

Transition preferences between approaches

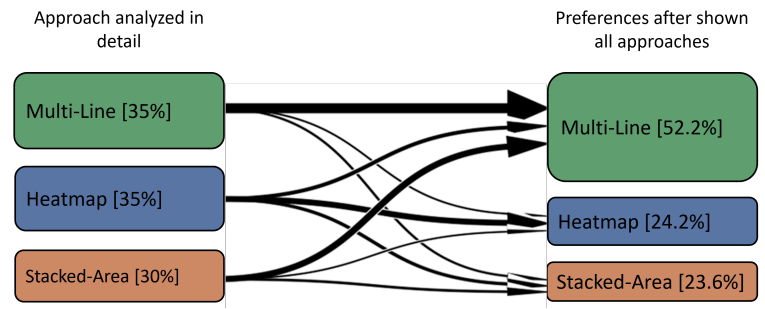

Figure 7: Preference transition after showing all three approaches to the participants.

\section{Conclusions}

In this paper, we investigated which visualization approaches are suitable for identifying the interdependencies of an MCS. The results showed that users are able to identify interdependencies and to use them to design a maintenance strategy for an MCS. Thus, visualization approaches seem suitable to overcome the challenge of identifying interdependencies. Specifically, the multi-line approach showed the best performance with respect to task performance (strategy validation and cost optimization). Moreover, the multi-line approach also clearly showed 
superiority based on subjective feedback (preference transitions). The multi-line and heatmap approach received better feedback regarding usability compared to stacked-area approach being more intuitive to use and easy to understand.

In this work, we evaluated the visualization approaches using a simple MCS (2-components). In the future, we plan to design a more complex MCS, which consists of a higher number of components, and evaluate the visualization approaches within this context. The complexity that can be introduced with the increase of the number of components is one crucial aspect that needs careful consideration, as it can easily affect the users' decisions while performing maintenance. Moreover, an evaluation of these approaches in terms of productivity (identifying perfect timing for maintenance) is required. So far we were able to evaluate approaches from the functional perspective. Next, we plan to analyze approaches based on the optimal solution. In this case, the recognition of the interdependencies at perfect timing (change point detection) is crucial for optimal maintenance. Moreover, in the future, we plan to integrate the approaches in a real DSS in the context of MCSs.

\section{Acknowledgements}

This work has been supported by the FFG, Contract No. 881844: Pro $^{2}$ Future is funded within the Austrian COMET Program Competence Centers for Excellent Technologies under the auspices of the Austrian Federal Ministry for Climate Action, Environment, Energy, Mobility, Innovation and Technology, the Austrian Federal Ministry for Digital and Economic Affairs and of the Provinces of Upper Austria and Styria. COMET is managed by the Austrian Research Promotion Agency FFG.

\section{References}

[1] Q. Wang, A. Farahat, K. Ristovski, H.-K. Tang, S. Serita, and C. Gupta, "What maintenance is worth the money? a data-driven answer," in 2018 IEEE 16th International Conference on Industrial Informatics (INDIN), pp. 284-291, IEEE, 2018.

[2] H. Lasi, P. Fettke, H.-G. Kemper, T. Feld, and M. Hoffmann, "Industry 4.0," Business \& information systems engineering, vol. 6, no. 4, pp. 239-242, 2014.

[3] S. Zhai, S. Achatz, M. Groher, J. Permadi, and G. Reinhart, "An empirical expert study on the status quo and potential of predictive maintenance in industry," in 2020 International Conference on Sensing, Diagnostics, Prognostics, and Control (SDPC), pp. 125-130, IEEE, 2020.

[4] M. Gashi and S. Thalmann, "Taking complexity into account: A structured literature review on multi-component systems in the context of predictive maintenance," in European, Mediterranean, and Middle Eastern Conference on Information Systems, pp. 31-44, Springer, 2019.

[5] A. Van Horenbeek and L. Pintelon, "A dynamic predictive maintenance policy for complex multi-component systems," Reliability Engineering \& System Safety, vol. 120, pp. 39-50, 2013.

[6] S. Thalmann, H. G. Gursch, J. Suschnigg, M. Gashi, H. Ennsbrunner, A. K. Fuchs, T. Schreck, B. Mutlu, J. Mangler, G. Kappl, et al., "Cognitive decision support for industrial product life cycles: A position paper," in Proceedings of the 11 th International Conference on Advanced Cognitive Technologies and Applications, pp. 3-9, 2019.

[7] K.-A. Nguyen, P. Do, and A. Grall, "Multi-level predictive maintenance for multi-component systems," Reliability engineering \& system safety, vol. 144, pp. 83-94, 2015.

[8] P. Carlshamre, K. Sandahl, M. Lindvall, B. Regnell, and J. N. och Dag, "An industrial survey of requirements interdependencies in software product release planning," in Proceedings Fifth IEEE International Symposium on Requirements Engineering, pp. 84-91, IEEE, 2001.

[9] R. Assaf, P. Do, P. Scarf, and S. Nefti-Meziani, "Wear rate-state interaction modelling for a multi-component system: Models and an experimental platform," IFAC-PapersOnLine, vol. 49, no. 28, pp. 232-237, 2016.

[10] A. F. Shahraki, A. Roy, O. P. Yadav, and A. P. S. Rathore, "Predicting remaining useful life based on instance-based learning," in 2019 Annual Reliability and Maintainability Symposium (RAMS), pp. 1-6, IEEE, 2019.

[11] M. C. O. Keizer, R. H. Teunter, and J. Veldman, "Joint condition-based maintenance and inventory optimization for systems with multiple components," European Journal of Operational Research, vol. 257, no. 1, pp. 209-222, 2017.

[12] J. Shen, A. Elwany, and L. Cui, "Reliability analysis for multi-component systems with degradation interaction and categorized shocks," Applied Mathematical Modelling, vol. 56, pp. 487-500, 2018.

[13] F. Chang, G. Zhou, C. Zhang, Z. Xiao, and C. Wang, "A service-oriented dynamic multi-level maintenance grouping strategy based on prediction information of multi-component systems," Journal of Manufacturing Systems, vol. 53, pp. 49-61, 2019.

[14] N. Rasmekomen and A. K. Parlikad, "Condition-based maintenance of multi-component systems with degradation state-rate interactions," Reliability Engineering \& System Safety, vol. 148, pp. 1-10, 2016.

[15] K. Gandhi, B. Schmidt, and A. H. Ng, "Towards data mining based decision support in manufacturing maintenance," Procedia Cirp, vol. 72, pp. 261-265, 2018.

[16] S.-j. Wu, N. Gebraeel, M. A. Lawley, and Y. Yih, "A neural network integrated decision support system for condition-based optimal predictive maintenance policy," IEEE Transactions on Systems, Man, and Cybernetics-Part A: Systems and Humans, vol. 37, no. 2, pp. 226-236, 2007.

[17] R. Yam, P. Tse, L. Li, and P. Tu, "Intelligent predictive decision support system for condition-based maintenance," The International Journal of Advanced Manufacturing Technology, vol. 17, no. 5, pp. 383-391, 2001. 
[18] Z. Li, Y. Wang, and K.-S. Wang, "Intelligent predictive maintenance for fault diagnosis and prognosis in machine centers: Industry 4.0 scenario," Advances in Manufacturing, vol. 5, no. 4, pp. 377-387, 2017.

[19] J. Suschnigg, B. Mutlu, G. Koutroulis, V. Sabol, S. Thalmann, and T. Schreck, "Visual exploration of anomalies in cyclic time series data with matrix and glyph representations," Big Data Research, p. 100251, 2021.

[20] M. Gashi, P. Ofner, H. Ennsbrunner, and S. Thalmann, "Dealing with missing usage data in defect prediction: A case study of a welding supplier," Computers in industry, vol. 132, p. 103505, 2021.

[21] Q. Sun, Z.-S. Ye, and N. Chen, "Optimal inspection and replacement policies for multi-unit systems subject to degradation," IEEE Transactions on Reliability, vol. 67, no. 1, pp. 401-413, 2017.

[22] S. Makridakis, "The forthcoming artificial intelligence (ai) revolution: Its impact on society and firms," Futures, vol. 90, pp. 46-60, 2017.

[23] J. Al-Kassab, Z. M. Ouertani, G. Schiuma, and A. Neely, "Information visualization to support management decisions," International Journal of Information Technology \& Decision Making, vol. 13, no. 02, pp. 407-428, 2014.

[24] B. Mutlu, M. Gashi, and V. Sabol, "Towards a task-based guidance in exploratory visual analytics," in Proceedings of the 54th Hawaii International Conference on System Sciences, p. 1466, 2021.

[25] S. Kim, I. Woo, R. Maciejewski, D. S. Ebert, T. D. Ropp, and K. Thomas, "Evaluating the effectiveness of visualization techniques for schematic diagrams in maintenance tasks," in Proceedings of the 7th Symposium on Applied Perception in Graphics and Visualization, pp. 33-40, 2010.

[26] X. Yang and S. Ergan, "Evaluation of various visualization forms for facility operation and maintenance," in ISARC. Proceedings of the International Symposium on Automation and Robotics in Construction, vol. 29, p. 1, IAARC Publications, 2012.

[27] S. Golovin, "Representation of replacement rules in the form of a matrix," Journal of Quality in Maintenance Engineering, 2016.

[28] A. Cachada, J. Barbosa, P. Leitño, C. A. Gcraldes, L. Deusdado, J. Costa, C. Teixeira, J. Teixeira, A. H. Moreira, P. M. Moreira, et al., "Maintenance 4.0: Intelligent and predictive maintenance system architecture," in 2018 IEEE 23rd international conference on emerging technologies and factory automation (ETFA), vol. 1, pp. 139-146, IEEE, 2018.

[29] J. C. Cheng, W. Chen, Y. Tan, and M. Wang, "A bim-based decision support system framework for predictive maintenance management of building facilities," in Proceedings of the 16th International Conference on Computing in Civil and Building Engineering (ICCCBE2016), 2016.

[30] J. C. Cheng, W. Chen, K. Chen, and Q. Wang, "Data-driven predictive maintenance planning framework for mep components based on bim and iot using machine learning algorithms," Automation in Construction, vol. 112, p. 103087, 2020.

[31] L. Wilkinson and M. Friendly, "The history of the cluster heat map," The American Statistician, vol. 63, no. 2, pp. 179-184, 2009.
[32] R. J. Pandolfi, D. B. Allan, E. Arenholz, L. Barroso-Luque, S. I. Campbell, T. A. Caswell, A. Blair, F. De Carlo, S. Fackler, A. P. Fournier, et al., "Xi-cam: a versatile interface for data visualization and analysis," Journal of synchrotron radiation, vol. 25, no. 4, pp. 1261-1270, 2018.

[33] N. Elmqvist, A. V. Moere, H.-C. Jetter, D. Cernea, H. Reiterer, and T. Jankun-Kelly, "Fluid interaction for information visualization," Information Visualization, vol. 10, no. 4, pp. 327-340, 2011

[34] A. Thudt, J. Walny, C. Perin, F. Rajabiyazdi, L. MacDonald, D. Vardeleon, S. Greenberg, and S. Carpendale, "Assessing the readability of stacked graphs," in Proceedings of Graphics Interface Conference (GI), 2016.

[35] W. S. Cleveland and R. McGill, "Graphical perception: Theory, experimentation, and application to the development of graphical methods," Journal of the American statistical association, vol. 79, no. 387, pp. 531-554, 1984.

[36] M. A. Borkin, A. A. Vo, Z. Bylinskii, P. Isola, S. Sunkavalli, A. Oliva, and H. Pfister, "What makes a visualization memorable?," IEEE transactions on visualization and computer graphics, vol. 19, no. 12, pp. 2306-2315, 2013.

[37] B. Mutlu, E. Veas, and C. Trattner, "Vizrec: Recommending personalized visualizations," $A C M$ Transactions on Interactive Intelligent Systems (TiiS), vol. 6, no. 4, pp. 1-39, 2016.

[38] C. Blome and M. Augustin, "Measuring change in quality of life: bias in prospective and retrospective evaluation," Value in Health, vol. 18, no. 1, pp. 110-115, 2015.

[39] Y. Weinstein and H. L. Roediger, "Retrospective bias in test performance: Providing easy items at the beginning of a test makes students believe they did better on it," Memory \& Cognition, vol. 38, no. 3, pp. 366-376, 2010.

[40] A. Kittur, E. H. Chi, and B. Suh, "Crowdsourcing user studies with mechanical turk," in Proceedings of the SIGCHI conference on human factors in computing systems, pp. 453-456, 2008.

[41] J. Brooke, "Sus: a "quick and dirty'usability," Usability evaluation in industry, vol. 189, 1996.

[42] H. B. Mann and D. R. Whitney, "On a test of whether one of two random variables is stochastically larger than the other," The annals of mathematical statistics, pp. 50-60, 1947.

[43] R. B. Patil, M. A. Patil, V. Ravi, and S. Naik, "Predictive modeling for corrective maintenance of imaging devices from machine logs," in 2017 39th Annual International Conference of the IEEE Engineering in Medicine and Biology Society (EMBC), pp. 1676-1679, IEEE, 2017.

[44] K. Nazemi, D. Burkhardt, E. Ginters, and J. Kohlhammer, "Semantics visualization-definition, approaches and challenges," Procedia Computer Science, vol. 75, pp. 75-83, 2015.

[45] D. Toker, C. Conati, G. Carenini, and M. Haraty, "Towards adaptive information visualization: on the influence of user characteristics," in International conference on user modeling, adaptation, and personalization, pp. 274-285, Springer, 2012.

[46] C. Conati and H. Maclaren, "Exploring the role of individual differences in information visualization," in Proceedings of the working conference on Advanced visual interfaces, pp. 199-206, 2008. 